\title{
Differentiation of retinal and nonretinal contributions to averaged evoked responses obtained with electrodes placed near the eyes
}

\author{
ROBERT G. EASON, LYNN FLOWERS, and MARTA OAKLEY \\ University of North Carolina, Greensboro, North Carolina 27412
}

\begin{abstract}
Recent visual evoked potential (VEP) studies suggest that during sustained voluntary attention, irrelevant or unattended visual stimuli may be filtered precortically. Testing of this hypothesis in humans requires knowledge of the origin of specific components of the VEP that are affected by selective attention. This study describes techniques and procedures for distinguishing between retinal and nonretinal components of VEPs recorded from electrodes placed strategically around the eyes and on the forehead. By visually stimulating one eye at a time while recording simultaneously from both, and by stimulating a given retinal area with the eyes fixated on the target at different angular deviations from the normal line of regard, components of the VEPs around the eyes can be distinguished as of retinal or nonretinal origin. The results are discussed in terms of volume conduction theory.
\end{abstract}

Visual evoked potential (VEP) studies conducted with humans in our laboratory (Eason, 1981; Eason, Harter, \& White, 1969; Eason, Oakley, \& Flowers, 1981) and by others (Van Voorhis \& Hillyard, 1977) indicate that early VEP components with latencies as short as $40-70 \mathrm{msec}$ can be modulated as a function of selective attention. Since these components probably arise from striate cortex (Creutzfeldt \& Kuhnt, 1967, 1973), their modulation may reflect centrifugally controlled filtering of certain kinds of unattended information prior to its arrival at the cortex (Eason, 1981; Eason et al., 1969). Such filtering conceivably could occur at all levels of the visual pathways, including the LGN, pulvinar, superior colliculus, and possibly the retina (Livingston, 1978), although opinions differ over the definitiveness of the evidence for the existence of centrifugal optic nerve fibers (Brindley, 1970; Hasselt, 1973; Livingston, 1978; Rodieck, 1973; Shortess, 1973).

In order to perform a direct test of the precortical filtering hypothesis in humans, those components of skin-recorded VEPs that reflect activity at various levels of the visual system must be delineated and taken into account. We have been conducting topographical studies for several years in an effort to establish the source of origin of such components.

This paper presents data from one of these studies

This study was presented at the annual meeting of the Psychonomic Society held in Philadelphia November 12-14, 1981. Address reprint requests and related correspondence to Robert G. Eason, Department of Psychology, University of North Carolina at Greensboro, Greensboro, North Carolina 27412. that focuses on the source of origin of components obtained with electrodes attached to the skin in close proximity to the eyes. A primary objective was to differentiate those components of the skin-recorded potentials of retinal origin from those of nonretinal origin. In attacking this problem, an experimental paradigm was employed that permitted the variation (with respect to the recording sites) of the location and orientation of hypothesized retinal dipole sources while holding extraretinal sources relatively constant.

The experimental paradigm entailed the simultaneous recording of averaged responses from electrodes homologously placed around the two eyes during stimulation of either the right or the left eye (i.e., monocular stimulation) with foveally centered light flashes under differing eye orientations. Monocular stimulation coupled with bilateral recordings provided one type of information pertaining to the source-of-origin issue; eye orientation provided another. The rationale for these manipulations follows.

The electrodes placed adjacent to the stimulated eye lie closer to the source of retinal activity than those placed adjacent to the unstimulated (occluded) eye. It follows from volume conduction principles (Rodieck, 1973) that electroretinographic (ERG) activity should be more pronounced for the stimulated eye electrode sites. Thus, the attainment of larger amplitude averaged response components from the stimulated than from the occluded eye electrode sites would constitute one type of evidence that the primary source of such activity is the retina. Extraretinal activity originating from more remote sources at approximately equal distances from the two eyes should have about the same impact on both 
eye electrode sites. Averaged response components of approximately equal magnitude obtained simultaneously from homologous sites would therefore constitute evidence for extraretinal sources of origin.

Manipulation of eye orientation, relative to the electrode sites, would seem to provide a particularly effective means of assessing whether averaged response components are of retinal origin, due to the fact that the longitudinal axis of a retinal dipole source of the stimulated eye would be dependent on the angular position of the eye in its orbit. It is known that the a-wave of the ERG is generated by the receptors (Armington, 1974; Brindley, 1970; Brown, 1968; Rodieck, 1973) and that the b-wave is generated by either the bipolar (Brown, 1968; Knave, Moller, \& Persson, 1972) or Müller cells (Dowling, 1970; Miller \& Dowling, 1970). Although some uncertainty may remain as to whether the b-wave arises from bipolar or Müller cells, or from both (Knave et al., 1972), it is the case that the longitudinal axis of the dipole sources arising from both the $a$ - and $b$-wave is oriented perpendicularly to the retinal surface (Brindley, 1970; Brown, 1968; Rodieck, 1973). The angular orientation of these dipole sources, relative to the recording electrodes, can be altered readily by rotating the eyes to various positions while holding constant the retinal area being stimulated. Based on volume conduction principles (Rodieck, 1973), maximal responses should be obtained from a given electrode site when the longitudinal axis of the retinal dipole is aligned with it;minimal responses should occur when the dipole axis lies at right angles to the electrode, and polarity inversions should be observed when there is a switch in the end of the dipole lying in closest proximity to the electrode. Such variations in averaged response components, as a function of eye orientation, would provide evidence for a retinal source of origin. In addition, they would serve as an index of the degree to which such activity is limited to a particular location on the retinal surface.

In addition to the manipulations described above, two stimulus parameters (stimulus size and background level) were manipulated in order to gain a rough indication of their effects on the skin-recorded responses. Such information was deemed relevant to our attention studies, wherein it is desirable to utilize stimuli of sufficient saliency to evoke measurable responses while avoiding unnecesarily high levels of saliency, which can be disruptive to the selective attention process (Eason, 1981; Eason et al., 1969). No attempt was made to establish precise quantitative relationships between these parameters and skin-recorded potentials, as has been done with corneally recorded ERGs (Armington, 1974).

\section{METHOD}

Two experiments were conducted. Four adults with normal or corrected vision participated in both experiments, in which averaged VEPs were obtained with electrodes placed homol- ogously about each eye during monoculat stimulation (one eye was covered with a patch). Eye orientation was varied along the vertical dimension in the first experiment, and along the horizontal dimension in the second. Stimulus size and background level was varied across experiments; flash luminance relative to background level was held constant.

In Experiment 1, averaged responses were obtained with electrodes placed above each eye (at standard positions $\mathrm{Fp}_{1}$ and $F p_{2}$ of the international electrode placement code) and immediately below each (designated LLE for the lower left eye position and LRE for the lower right eye position). The subject fixated the center of the stimulus display with the eyes (1) rotated upward $20 \mathrm{deg}$, (2) straight ahead, or (3) rotated downward $30 \mathrm{deg}$. The evoking stimuli consisted of 18-deg circular flashes of 10 microsec duration. The flashes contained a pattern of black and white checks, each check subtending a visual angle of $30 \mathrm{~min}$. Subjects were instructed to fixate the center of the stimulus display area. The brightness of the flashes was approximately $4 \mathrm{log}$ units above a background luminance level of $.01 \mathrm{~mL}$. Four averaged response replications were obtained under each experimental condition from each subject in counterbalanced order.

In Experiment 2, responses were obtained with electrodes placed at the internal and external canthi of each eye during monocular fixation of the center of the stimulus display with the uncovered eye rotated (1) medially $30 \mathrm{deg}$ or (2) laterally $40 \mathrm{deg}$. The yoked orientations of the covered eye were, of course, the inverse of these orientations. Evoking stimuli consisted of 6.5-deg circular black and white flashed checkered patterns, the brightness of which was about $4 \mathrm{log}$ units above a background luminance level of $3.0 \mathrm{~mL}$. Check size was the same as in Experiment 1. Two averaged response replications were obtained in counterbalanced order under each experimental condition for each subject.

The following procedures were common to both experiments. A Grass photostimulator illuminated the stimulus display area, which was located at a distance of $60 \mathrm{~cm}$ from the stimulated eye. Flashes occurred at irregular intervals ranging from 1 to $3 \mathrm{sec}$, the average interval being $1.3 \mathrm{sec}$. Body and head orientation was held constant with the aid of a headrest. To achieve the desired eye orientation in a given run, the display was positioned at the appropriate location and the subject was instructed to rotate the eyes without moving the body or head, so as to fixate its center. Responses were amplified with Grass EEG preamplifier units coupled to a Model 7 polygraph. The $50 \%$ amplitude lower and upper bandpass filters were set at 1 and $35 \mathrm{~Hz}$. The amplified potentials were averaged with a TMC signal averager, Model B; each average was based on 100 stimulus presentations. All recordings were monopolar, the right earlobe serving as a reference. This reference was chosen after having established in a pilot study on one of the subjects that the choice of earlobe (right or left) had no discernible effect on the magnitude or waveform of early response components (i.e., those occurring within the first $200 \mathrm{msec}$ following presentation of the stimulus flashes) obtained at the active electrode sites. The subjects were grounded through an electrode attached to the left earlobe. While recording, "white" noise was channeled into the subjects' electrically shielded room to mask extraneous sounds.

\section{RESULTS}

\section{Experiment 1}

The results obtained in the first experiment are summarized pictorially in Figure 1. The eye diagrams (top row of the figure) indicate the angular orientation, relative to the electrode sites, of the longitudinal axis of a hypothesized retinal dipole source activated by foveally 


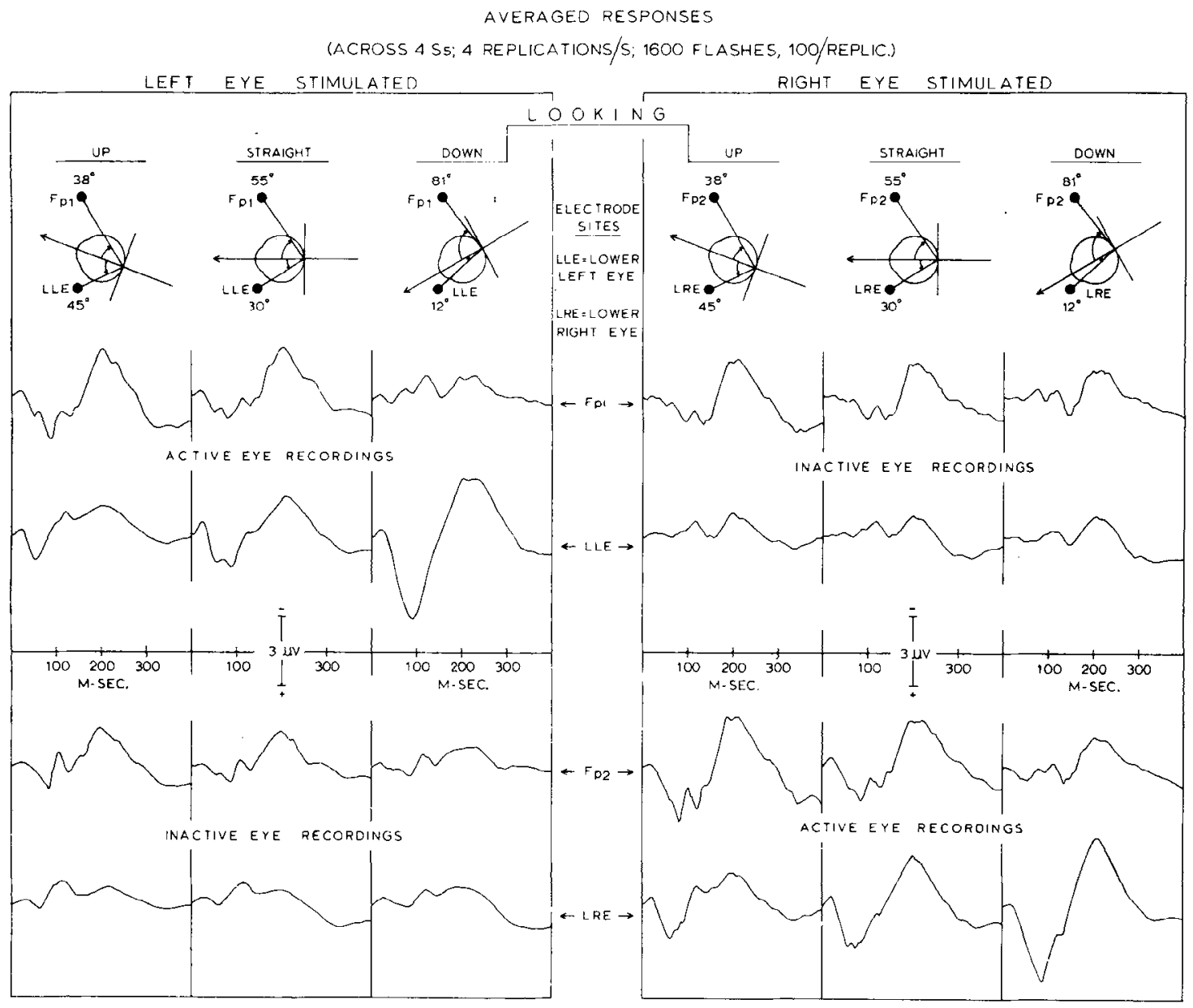

Figure 1. Group averaged responses obtained from electrodes placed at homologous locations above and below the two eyes (a) during stimulation of either the left or right eye and (b) with the eyes rotated upward, straight ahead, or downward. The eye diagrams depict the location of the recording electrodes relative to the longitudinal axis of a hypothesized dipole source originating from within the stimulated eye. Responses shown in the upper left quadrant were obtained with electrodes placed about the stimulated left eye; those in the lower left quadrant were simultaneously obtained from the unstimulated right-eye electrodes. Responses in the lower right quadrant were obtained from electrodes placed about the stimulated right eye; those of the upper right quadrant were simultaneously obtained from the unstimulated left-eye electrodes. Negativity is up. See text for further details.

centered stimulation for each eye orientation. Results obtained for left-eye stimulation are shown on the left; those for right-eye stimulation are shown on the right. Left-eye recordings are shown in the upper two rows; right-eye recordings are shown in the lower two. It should be noted that the tracings in the lower left quadrant were recorded from the right-eye electrode sites (labeled "inactive" eye recordings) when the left eye was stimulated. Those in the upper right quadrant were recorded from the left-eye electrode sites (also labeled "inactive" eye recordings) when the right eye was stimulated.

Each tracing is the combined average of 16 separate averaged potentials obtained over four replications for each of the four subjects. Each composite average is based on a total of 1,600 stimulus presentations, 100 per individual average. The waveforms of the composite averages closely resembled the individual averages for each subject.

Description of components. Three components contained in the composite responses obtained from the stimulated eye (upper left and lower right quadrants) can readily be identified as ERG components on the basis of their shape, latency, and polarity. A small negative component (negativity is up) with a peak latency of approximately $20 \mathrm{msec}$ is the a-wave; a larger positive deflection with a peak latency of about 60 . $80 \mathrm{msec}$ is the b-wave, and a late negative potential with a peak latency of approximately $200 \mathrm{msec}$ is generally referred to as the afterpotential (Armington, 
1974). Small wavelets can be observed "riding" on the primary ERG components, the most prominent of which is a negative deflection with a peak latency of approximately $100 \mathrm{msec}$. Although not as apparent in the composite tracings, relatively large deflections were sometimes observed to "ride" on both the b-wave and afterpotential of the individual averaged potentials obtained at the upper electrode sites $\left(\mathrm{Fp}_{1}\right.$ and $\left.\mathrm{Fp}_{2}\right)$.

The b-wave, afterpotential, and 100-msec deflection, along with several other small deflections, can be observed in the responses obtained from electrodes located about the unstimulated eye (lower left and upper right quadrants). The $\mathrm{b}$-wave and afterpotential components tend to be much smaller than those obtained from the stimulated eye electrodes, and they may be viewed as "farfield" recordings of the ERG of the stimulated eye. An a-wave generally was not discernible in the individual averaged responses, nor is it clearly apparent in the composite tracings.

Quantitative analyses. To quantify the data, the evoked responses obtained from each subject under each experimental condition were first averaged across replications. This produced a single averaged response per subject per condition, each being based on a total of 400 stimulus presentations. The peak-to-peak height of the b-wave (vertical distance from the peak of the a-wave to the peak of the b-wave) and afterpotential (vertical distance from the peak of the $b$-wave to the peak of the afterpotential) was then measured for each such response, yielding four scores (one for each subject) for each dependent variable per experimental condition. Three-way analyses of variance ( 2 by 3 by 4 ) involving (1) eye stimulated or electrode location, (2) eye orientation, and (3) subjects, with a total of 23 degrees of freedom, were performed on each of the two measures. No attempt was made to quantify the a-wave because of its small size.

Monocular stimulation effects (flashed vs. occluded eye). The effects of stimulation of a given eye on the responises recorded simultaneously from homologous locations of the two eyes are revealed by several different comparisons in Figure 1. First, a comparison of the two rows of tracings in the upper left quadrant to the appropriate counterpart (with respect to eye orientation and electrode position) in the upper right quadrant reveals that greater responses were obtained from the left-eye electrode locations when that eye was stimulated ( $\mathrm{Fp}_{1}$ and LLE labeled "active") than when it was not ( $\mathrm{Fp}_{1}$ and LLE labeled "inactive"). The observed differences were found to be statistically significant $\left[F(1,15)=8.45, p<.05\right.$, for $F p_{1}$ b-wave; $F(1,15)=$ $5.90, \mathrm{p}<.05$, for the $\mathrm{Fp}_{1}$ afterpotential; $F(1,15)=$ $14.03, \mathrm{p}<.01$, for the LLE $b$-wave; $F(1,15)=15.12$, $\mathrm{p}<.01$, for the LLE afterpotential]. Second, a comparison of the lower two quadrants reveals a similar effect for the right-eye recordings $[F(1,15)=4.54, p<.05$, for the $F p_{2}$ b-wave; $F(1,15)=9.32, p<.01$, for the $\mathrm{Fp}_{2}$ afterpotential; $\mathrm{F}(1,15)=19.11, \mathrm{p}<.01$, for the
LRE b-wave; $F(1,15)=22.71, p<.01$, for the LRE afterpotential]. Third, a comparison of the tracings in the upper left quadrant to the appropriate counterpart in the lower left quadrant reveals a tendency for responses obtained from above and below the stimulated left eye to be greater than those simultaneouly obtained from homologous sites of the unstimulated right eye. The apparent differences, although in the expected direction, were not statistically significant for the upper electrode locations $\left(F p_{1}\right.$ vs. $\left.F p_{2}\right)$; however, they were significant for the lower locations (LLE vs. LRE) $[F(1,15)=7.63, p<.05$, for the b-wave; $F(1,15)=$ $20.63, p<.01$, for the afterpotential]. A similar comparison of the upper right to the lower right quadrant also reveals a tendency for the responses obtained in close proximity to the stimulated right eye to be greater than those simultaneously obtained from homologous locations for the unstimulated left eye. These effects were statistically significant $[F(1,15)=9.22, p<.01$, for the b-wave amplitude difference between $F_{p_{1}}$ and $\mathrm{Fp}_{2} ; \mathrm{F}(1,15)=9.96, \mathrm{p}<.01$, for the afterpotential amplitude difference between $F p_{1}$ and $F p_{2} ; F(1,15)=$ $28.06, p<.01$, for the $b$-wave difference between LLE and LRE; $F(1,15)=20.50, p<.01$, for the afterpotential difference betwen LLE and LRE].

Eye orientation effects. A between-column comparison within the upper left quadrant of Figure 1 reveals the effects of eye orientation on responses obtained from recording sites above and below the stimulated left eye; a similar comparison within the lower right quadrant reveals corresponding effects for the stimulated right eye. For both eyes, when stimulated, the upper site recordings were largest when the eyes were rotated upward, of intermediate size when oriented straight ahead, and smallest when rotated downward. Variance analyses, collapsed across $F p_{1}$ and $F p_{2}$, revealed that these effects were statistically significant $[F(2,15)=$ $12.56, p<.01$, for the $b$-wave; $F(2,15)=15.16, p<.01$, for the afterpotential].

The effect of eye orientation on the amplitude of the major response components also is revealed by comparing the upper $\left(F p_{1}\right)$ to the lower (LLE) electrode tracings for the stimulated left eye (upper left quadrant) and the upper $\left(\mathrm{Fp}_{2}\right)$ to the lower (LRE) tracings for the stimulated right eye (lower right quadrant). An inverse relationship between the amplitude of the responses obtained from the upper and lower electrode sites with respect to eye orientation clearly manifests itself for each eye when stimulated. An analysis of variance was performed after collapsing data across the two eyes, when stimulated, and across response components (i.e., across the b-wave and afterpotential). The analysis revealed a statistically significant interaction between electrode location (upper vs. lower) and eye orientation $[F(2,15)=4.84, p<.05]$.

A comparison of the stimulated eye recordings to the eye diagrams of Figure 1 reveals the existence of an inverse relationship between the magnitude of the major 
components of the averaged responses and the degree of angular displacement between the recording electrode and the longitudinal axis of a hypothesized retinal dipole.

\section{Experiment 2}

Results obtained in the second experiment are summarized in Figure 2. The eye diagrams in the left portion of the figure indicate the angular orientation, relative to the electrode sites, of the longitudinal axis of the hypothesized retinal dipole source activated by foveally centered stimulation for each eye orientation. The top two diagrams apply to left-eye stimulation; the bottom two apply to right-eye stimulation. Recordings obtained during left-eye stimulation are shown in the left quadrants; those for right-eye stimulation are shown in the right quadrants. The upper quadrants contain responses obtained with electrodes placed at the canthi of the stimulated eye. The lower quadrants show responses simultaneously obtained from homologous sites of the unstimulated eye.
Each tracing is the composite average of eight separate averaged potentials obtained over two replications for each of the four subjects and is based on a total of 800 stimulus presentations ( 100 per replication per subject).

Description of components. As in Experiment 1, the a- and b-waves of the ERG, as well as the afterpotential, can be identified in the composite averages obtained for the stimulated eye. However, the polarity of each of these components depends on the experimental condition under which the recordings were obtained, as discussed below. In addition to the basic ERG components, the wavelets described in Experiment 1 can be observed in the recordings obtained from both stimulated and unstimulated eye sites, being most noticeable for the unstimulated eye (lower quadrants). The most prominent of these was again a negative deflection with a peak latency of approximately $100 \mathrm{msec}$.

Quantitative analyses. As in Experiment 1, VEPs obtained from each subject under each experimental
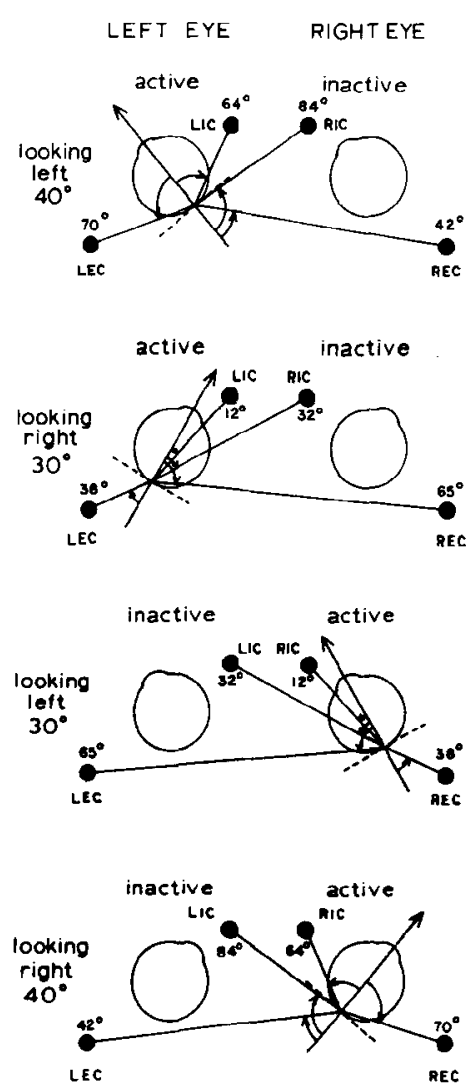

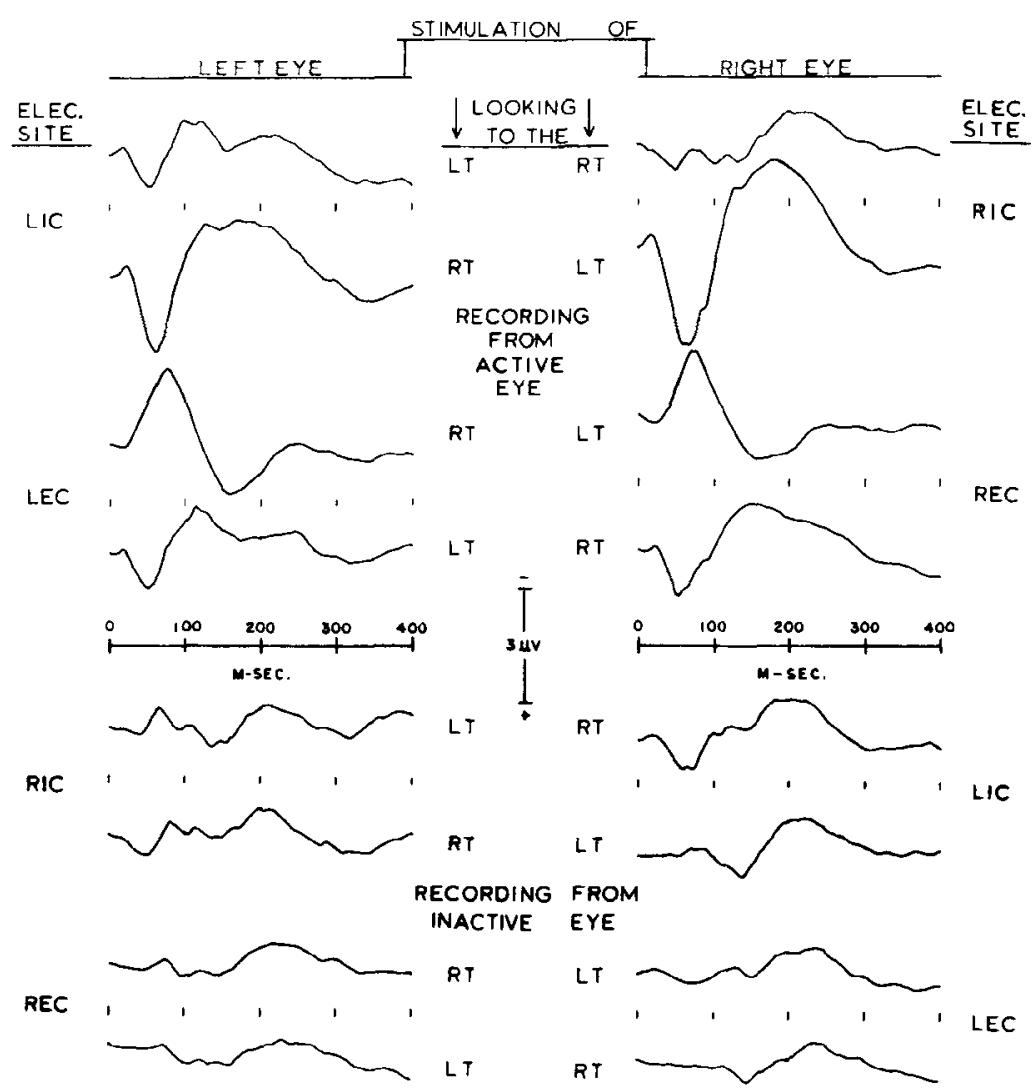

Figure 2. Group averaged responses obtained from electrodes placed at the external and internal canthi of each eye during stimulation of each eye separately, and with the eyes rotated either to the left or to the right. The eye diagrams show the electrode locations relative to the longitudinal axis of a hypothesized retinal dipole originating from within the stimulated eye. The top two eye diagrams apply to left-eye stimulation; the lower two apply to right-eye stimulation. The averaged responses in the upper left quadrant were obtained from the stimulated left-eye electrodes; those in the upper right quadrant were obtained from the stimulated right-eye electrodes. Responses in the lower left quadrant were obtained from the unstimulated right-eye electrodes; those in the lower right were obtained from the unstimulated left-eye electrodes. Negativity is up. See text for further details. 
condition were averaged across replications, resulting in a single averaged tracing (based on 200 stimulus presentations) per condition per subject. Peak-to-peak measurements of the b-wave and afterpotential yielded four scores for each dependent variable, one per subject for each experimental condition. Individual $t$ tests were performed on each comparison discussed below. Even though there were only 3 degrees of freedom associated with each $t$ test (number of subjects minus 1), the tests were sufficiently powerful to demonstrate statistical significance due to the robustness of the effects of the experimental manipulations.

Monocular stimulation effects (flashed vs. occluded eye). Consistent with the results of Experiment 1, the b-wave and afterpotential components of the responses obtained from the stimulated eye electrode sites (upper quadrants of Figure 2) were of much greater magnitude than those obtained from the unstimulated eye sites (lower quadrants). The $t$ tests performed on the amplitude difference scores between the internal canthus recordings of the two eyes when stimulated vs. when unstimulated, after having averaged the difference scores across the two eye orientations, revealed significant effects for each of the quantified response components $[t(3)=7.58, p<.01$, for the b-wave; $t(3)=6.24$, $\mathrm{p}<.01$, for the afterpotential]. Similar tests performed on the external canthus recordings also revealed significant effects $[\mathrm{t}(3)=9.59, \mathrm{p}<.01$, for the $\mathrm{b}$-wave; $t(3)=10.58, p<.01$, for the afterpotential]. In some instances, there were polarity reversals between the stimulated and unstimulated eye recordings.

Eye orientation effects. A number of comparisons in Figure 2 reveal pronounced effects of eye rotation on the amplitude and polarity of the responses obtained from the stimulated eye electrodes (upper quadrants). First, the amplitudes of both the b-wave and afterpotential of the internal canthus responses (LIC and RIC) were greater when the stimulated eye was rotated medially (second row) than when it was rotated laterally (first row) $[t(3)=3.85, p<.05$, for the LIC b-wave; $\mathrm{t}(3)=3.20, \mathrm{p}<.05$, for the LIC afterpotential; $\mathrm{t}(3)=$ $5.24, \mathrm{p}<.05$, for the RIC b-wave; $\mathrm{t}(3)=7.07, \mathrm{p}<.01$, for the RIC afterpotential]; none of the components tended to invert in polarity. These results are consistent with predictions based on the hypothesized retinal dipoles depicted for the LIC and RIC electrode sites in the eye diagrams of Rows 1 and 3. These diagrams reveal that for medial orientations of the stimulated eye, the angular displacement between the internal canthus electrodes and the longitudinal axis of the hypothesized retinal dipole was a relatively favorable $12 \mathrm{deg}$; whereas for the lateral orientations, the angular displacement was a relatively unfavorable $64 \mathrm{deg}$. For both orientations, the internal canthus electrodes maintained an anterior position relative to the dipole source. This is consistent with the observed absence of a polarity inversion.

Second, with the stimulated eye rotated medially, the polarity of the major response components obtained from the internal canthus electrodes (LIC and RIC of Row 2) was of opposite polarity to those obtained from the external canthus electrodes (LEC and REC of Row 3 ). The inversion occurred consistently for every subject, and the differences in amplitude for both the b-wave and afterpotential, taking the direction of the deflections into consideration, were statistically significant in every case $[t(3)=7.39, p<.01$, for the $b$-wave difference between LIC and LEC; $t(3)=11.96$, $\mathrm{p}<.01$, for the afterpotential difference between LIC and LEC; $t(3)=6.71, p<.01$, for the $b$-wave difference between RIC and REC; $t(3)=6.08, p<.01$, for the afterpotential difference between RIC and REC]. A comparison of these results to the eye diagrams in Rows 2 and 3 indicates that the effects are consistent with the predictions to be made on the basis of the location of the recording electrodes relative to the hypothesized retinal dipoles. The internal canthus electrodes occupy a position anterior to the dipole source at an angular displacement of $12 \mathrm{deg}$, whereas the external canthus electrodes are positioned posteriorly to the source at an angular displacement of $38 \mathrm{deg}$. Such an arrangement would be expected to produce the polarity inversion manifested between the tracings in Rows 2 and 3 of Figure 2.

Finally, a polarity inversion occurred in the external canthus recordings when the stimulated eye shifted from a medial position (third row) to a lateral orientation (fourth row). The polarity inversion occurred consistently for all subjects. The amplitude differences for both the $b$-wave and afterpotential, taking the direction of the deflections into consideration, were statistically significant $[\mathrm{t}(3)=9.71, \mathrm{p}<.01$, for the LEC b-wave; $\mathrm{t}(3)=$ $8.77, \mathrm{p}<.01$, for the LEC afterpotential; $\mathrm{t}(3)=6.09$, $p<.01$, for the REC b-wave; $t(3)=8.83, p<.01$, for the REC afterpotential]. In reference to the eye diagrams, this inversion is consistent with a shift in the location of the external canthus electrodes from an anterior to a posterior position, relative to the dipole source, when the activated eye is changed from a lateral to a medial orientation (compare LEC sites in the two upper eye diagrams; aiso the REC sites in the two lower diagrams).

Although the a-wave was not quantified, due to its small size, the inversions described above for the $b$-wave and afterpotential also can be discerned in the a-wave of the tracings shown in Rows 2,3, and 4 of the upper quadrants.

Shifts in eye position had little effect on the amplitude and polarity of the responses recorded from the unstimulated eye (lower quadrants), and no statistical tests were performed on these responses, since they obviously did not differ significantly.

\section{Across-Experiment Comparisons: \\ Stimulus Parameter Effects}

Since different electrode locations and different eye orientations were used in the two experiments, a precise analysis of the effects of stimulus size and background 
level on the magnitude of the averaged response components was not made. However, certain recordings in Figure 1 were compared to those in Figure 2, wherein the degree of angular displacement between the longitudinal axis of a hypothesized retinal dipole source and electrode location was held constant. One such comparison involved the left-eye response obtained from the LLE electrode site with the eyes oriented downward in Figure 1 (Row 2, Column 3 of the upper left quadrant) and the right-eye response obtained from the RIC electrode site with the eyes looking to the left in Figure 2 (Row 2 of the upper right quadrant). In both instances, the recording electrode was located anterior to a hypothesized retinal dipole source at an angular displacement of $12 \mathrm{deg}$. The responses obtained in the two experiments were approximately equal in magnitude. This seems reasonable, since the evoking stimulus in each of the two experiments was approximately $4 \mathrm{log}$ units above its respective background (i.e., flash intensity, relative to background, was held constant).

An analogous comparison between the LRE recording in the lower right quadrant of Figure 1 (Row 2, Column 3) and the LIC recording in the upper left quadrant of Figure 2 (Row 2) reveals that the response obtained in the first experiment was slightly larger than that obtained in the second.

A comparison of other response pairs similarly matched with respect to angular displacement also revealed that responses were approximately equal in magnitude. Thus, variations in background level from dark $(.01 \mathrm{~mL})$ to twilight $(3.0 \mathrm{~mL})$ had little or no attenuating effect on the ERG, provided that the intensity of the evoking stimulus, relative to background level, was held constant.

The effect of stimulus size also appears to have been minimal, perhaps because of the relatively high intensity of the stimulus flashes in relation to their respective backgrounds and/or to the relatively large size of the stimuli used in each of the two experiments.

\section{DISCUSSION}

In general, the results of this study indicate that the retina is the major source of electrical activity contributing to averaged responses obtained with electrodes placed about the eyes. The larger responses obtained in both experiments from the stimulated eye electrode sites, compared to those obtained from homologous sites for the unstimulated contralateral eye, support this conclusion. The larger responses obtained from a given eye when stimulated, compared to the magnitude of the responses obtained from that same eye when unstimulated (i.e., the contralateral eye is stimulated), provide additional support. The eye orientation effects provide further support by virtue of the fact that the observed changes in amplitude and/or polarity of the responses obtained from the various electrode sites were consistent with the predictions derivable from the hypothesized retinal dipole models shown in Figures 1 and 2. Finally, the major components of the averaged responses obtained with skin electrodes placed in close proximity to the stimulated eye match the descriptions of the a-wave, b-wave, and afterpotential of the human ERG recorded with corneal electrodes (Armington, 1974), providing further evidence of their retinal origin.

\section{Extraretinal Potentials}

Considering that ERGs are recorded routinely in clinical situations, using skin electrodes and an averaging computer (Harden, Picton-Robinson, Bradshaw, \& Pampiglione, 1980;Sulg, Hokkanen, Siivola, \& Reunanen, 1980), it is not surprising that the major results of this study should point to the retina as the primary source of the most prominent deflections. However, for a number of reasons, one should not assume that all averaged activity obtained with electrodes placed in close proximity to the eyes is of retinal origin. First, it should be noted that a much higher amplification factor is required to record the averaged ERG with skin electrodes than with corneal electrodes. The largest components observed in our experiments did not exceed $10 \mathrm{mV}$, whereas the corneally recorded b-wave is known to reach several hundred microvolts (Armington, 1974). Based on Rodieck's (1973) analysis of volume conduction, the greatly diminished amplitude of the skin-recorded ERG may be attributed to increased resistance encountered in the flow of current from the eye to the extraorbital areas. Second, while the extraorbital skin areas constitute less favorable electrode locations for recording the ERG than does the cornea, they are more favorable locations for recording nonretinal potentials arising from nearby neural or myogenic sources, due to a reduction in resistance to volume conduction current flow. The combined consequences of using skin electrodes, rather than corneal ones (i.e., higher amplification, higher resistance to current flow from retinal sources, and lower resistance to current flow from neural and myogenic sources) greatly increases the probability of recording nonretinal along with retinal activity.

The most consistently occurring wave observed in the present study, which appears to be of nonretinal origin, was a 100 -msec negative deflection that "rode" on the afterpotential of the ERG. Its nonretinal origin is suggested by the finding that the wave was just as prominent for unstimulated as for stimulated eye recordings. Possible sources of origin are time-locked myogenic responses arising from frontalis and/or the eyelid muscles, electrooculographic (EOG) fluctuations generated by minute flash-induced reflex eye movements, timelocked neural activity originating in visual structures central to the retina, or some combination of the three.

With respect to the myogenic possibility, it is known that reflex blinks to both visual and auditory stimulation can occur within $100 \mathrm{msec}$ or less (Bixler, Bartlett, 
\& Lansing, 1967; Bohlin \& Graham, 1977) and that myogenic potentials may appear in frontal scalp recordings to diffuse light flashes (Allison, Matsumiya, Goff, $\&$ Goff, 1977). Since the eyes tend to roll upward when the lids close (Mulholland \& Evans, 1965), the 100-msec deflection could reflect the algerbraic summation of both myogenic and EOG contributions.

The polarity of the $100-\mathrm{msec}$ deflection recorded at various electrode locations should serve as an important cue as to which of the above three possibilities contributed to its development. Since the electrodes placed above and below the eyes are on opposite sides of the eyelid, averaged electromyographic (EMG) activity recorded simultaneously from these two locations due to eye blinks should be of opposite polarity. Also, eye movement-induced changes in the EOG recorded simultaneously from the upper and lower electrode sites, as well as from the nasal and temporal canthal locations, should be of opposite polarity, since a reciprocal relationship exists between the angular distance of oppositely placed electrodes and the corneoretinal potential dipole axis. To verify empirically that recordings obtained from opposite sides of the eye are of inverted polarity, we obtained averaged responses from above and below the eye of one subject who voluntarily blinked or rolled the eyes upward each time a stimulus flash was presented. Biphasic waves generated at each electrode site to each type of motor responses were observed to be of opposite polarity.

Inspection of the 100-msec deflection in both Figures 1 and 2 reveals that its polarity was always negative regardless of the electrode position from which a recording was obtained. This observation held for both the active and inactive eye recordings. It is concluded, therefore, that the $100-\mathrm{msec}$ deflection could not have been due to either involuntary eye hlinks or eye movements. The absence of evidence of minute eye movement contamination of recordings in this study is consistent with the results of an earlier study by Eason (1981), which indicated no time-locked movements in averaged EOGs recorded at external canthal electrode sites to peripherally presented stimulus flashes.

The possibility remains that time-locked frontalis and/or other scalp muscle activity may have contributed to the development of the 100 -msec deflection, since EMG activity is clearly more evident in recordings obtained with electrodes placed above the eyes than with electrodes placed below the eyes. If time-locked EMG activity was a contributor, the amplitude of the deflection obtained at the upper electrode sites might be expected to be greater than that obtained at lower sites due to the closer proximity of the upper sites to frontalis muscle. A comparison of amplitudes of the 100-msec deflection for upper and lower sites in Figure 1 reveals little, if any, difference, casting considerable doubt on whether the deflection is of myogenic origin.
It appears unlikely that the deflection is due to scalp muscle twitches, eye blinks, or eye movements. The remaining possibility is that it reflects neural activity originating in areas lying central to the retina. Whether such neural activity is registered entirely by the electrodes placed about the eyes, with no change occurring at the earlobe reference electrode site, remains to be determined.

\section{Stimulus Parameter Effects}

The primary purpose for increasing background level and reducing stimulus size in the second experiment was to establish whether stable, repeatable, and measurable ERGs could be obtained with skin electrodes under stimulus conditions less salient than those in the first experiment. The results of Experiment 2 clearly demonstrated that averaged ERGs can indeed be obtained to relatively small stimuli under twilight background conditions. To the extent that valid comparisons can be made between the two experiments with respect to variations in background level and stimulus size, the results seem compatible with those reported in parametric studies using corneal electrodes (Armington, 1974).

\section{Stray Light}

Although some degree of retinal light scatter was unquestionably present when stimulus flashes were presented to the eye (Armington, 1974; Boynton, 1953; Boynton \& Riggs, 1951), the eye manipulation effects seemed to indicate that relatively localized retinal activity was the principal contributor to the ERG activity registered at a given electrode site. The eye orientation effects are consistent with an earlier observation by Crampton and Armington (1955) that the amplitude of the $b$-wave varies inversely with the distance from the retinal area stimulated and the recording electrode. They concluded that the $b$-wave could not be due solely to stray light.

\section{Efficacy of Electrode Location}

Although all electrodes placed in close proximity to the stimulated eye proved to be effective in detecting the ERG, with the aid of an averaging computer, certain locations were found to be more effective than others. Equating for eye orientation, larger responses were obtained from lower than from upper eye sites. However, the upper sites were more sensitive to variations in orientation. Recall that both the $b$-wave and afterpotential of upper eye recordings were significantly affected by vertical variations in eye orientation, whereas for lower eye recordings, only the b-wave of the left eye was significantly affected. It appears that the signal reaching the lower eye electrodes via volume conduction traversed a more diffusely defined route than did that reaching the upper electrode sites, thereby causing more "smearing" of the orientation of the retinal dipole giving rise to the signal. 
With respect to the electrode positions used in Experiment 2, the internal canthus location tended to be somewhat more sensitive for detecting ERG activity than was the external canthus site, given matched angular displacement of eye orientation relative to the two electrode sites. Both canthal locations were very sensitive to variations in eye orientation along the horizontal axis.

We make the following conclusions: (1) When locus of retinal stimulation is held constant, Positions $F p_{1}$ (for left-eye stimulation) and $F_{p_{2}}$ (for right-eye stimulation) are especially sensitive to vertical variations in eye orientation and, therefore, are favorable locations for registering variations in relatively localized retinal activity along the vertical axis. (2) The internal and external canthal locations are favorable sites for recording variations in localized retinal activity along the horizontal meridian. (3) Equating for eye orientation, the lower eye position is a sensitive site for recording diffuse ERGs from the stimulated eye, but relatively poor for detecting variations in localized retinal dipole activity. (4) The upper eye sites are relatively vulnerable to myogenic artifacts; lower eye and canthal sites are relatively free of such artifacts.

\section{Implications}

With electrodes placed about the eyes, the results obtained with monocular stimulation while recording binocularly and with varying eye orientations demonstrate the utility of these manipulations in delineating retinal from nonretinal potentials. Such manipulations should prove useful in differentiating between retinal and nonretinal influences on VEPs obtained in topographical studies involving multiple electrode placements on the scalp. The manipulations also may prove to be beneficial in certain attention studies involving multiple electrode placements for the purpose of establishing where in the visual system certain kinds of unattended or irrelevant information are filtered. Another potential use is in clinical work involving making decisions as to whether certain anomalies seen in scalp VEP recordings are due primarily to retinal dysfunction or to more centrally located neural difficulties.

\section{REFERENCES}

Allison, T., Matgumiya, Y., Gorf, G. D., \& Gorf, W. R. The scalp topography of human visual evoked potentials. Electroencephalography and Clinical Neurophysiology, 1977, 12, 185197.

Anminaton, J. C. The electroretinogram. New York: Academic Press, 1974.

Bixler, E. O., Barteett, N. R., \& Lansing, R. W. Latency of the eye blink reflex and stimulus intensity. Perception \& Psychophysics, 1967, 2, 559-560.

Bohi,in, G., \& Graham, F. K. Cardiac deceleration and reflex blink facilitation. Psychophysiology, 1977, 14, 423-430.
Boynton, R. M. Stray light and the human electroretinogram. Journal of the Optical Society of America, 1953, 43, 442-449.

Boynton, R. M., \& Rigas, L. A. The effect of stimulus area and intensity upon the human retinal response. Journal of Experimental Psychology, 1951, 42, 217-226.

Brindley, G. S. Physiology of the retina and visual pathway (2nd ed.). London: Arnold, 1970.

Brown, K. T. The electroretinogram: Its components and their origins. Vision Research, 1968, 8, 633-677.

Champton, G. H., \& Arminoton, J. C. Area-intensity relation and retinal location in the human electroretinogram. American Journal of Physiology, 1955, 181, 47.53.

Creutzreldt, O. D., \& Kuhrt, U. The visual evoked potential: Physiological, developmental and clinical aspects. In W. Cobb \& C. Morocutti (Eds.), The evoked potentials. Amsterdam: Elsevier, 1967.

Cneutzeredt, O. D., \& Kuhnt, U. Electrophysiology and topographical distributions of visual evoked potentials in animals. In R. Jung (Ed.), Handbook of sensory physiology. Berlin: Springer-Verlag, 1973.

Dowling, J. E. Organization of vertebrate retinas. Investigative Ophthalmology, 1970, 9, 655-680.

EAson, R. G. Visual evoked potential correlates of early neural filtering during selective attention. Bulletin of the Psychonomic Society, 1981, 18, 203-206.

Eason, R. G., Harter, M. R., \& White, C. T. Effects of attention and arousal on visually evoked cortical potentials and reaction time in man. Physiology \& Behavior, 1969, 4, 283-289.

Eabon, R. G., OAxLey, M., \& Flowers, L. Selective attentioninduced centrifugal influences on the human retina. Society for Neuroscience Abstracts, 1981, 7, 659.

Hatden, A., Picton-Robingon, N., Bandbhaw, K., \& PAmplalione, G. Ten years' experience of ERO/VEP/EEG studies on visual disorder in paediatrics. In C. Barber (Ed.), Evoked potentials. Baltimore, Md: University Park Press, 1980.

Hassalt, P. vaN. The centrifugal control of retinal function: A review. Ophthalmological Research, 1973, 4, 298-320.

Knave, B., Moller, A., \& Perseon, H. E. A component analysis of the electroretinogram. Vision Research, 1972, 12, 1669-1684.

Livinaston, R. B. Sensory processing, perception, and behavior. New York: Raven Press, 1978.

Mille R, R. F., \& Dowling, J. E. Intracellular responses of the Müler (glial) cells of mudpuppy retina: Their relation to b-wave of the electroretinogram. Journal of Neurophysiology, 1970, 33, 323-341.

Mulholland, T., \& Evans, C. R. An unexpected artifact in the human electroencephalogram concerning the alpha rhythm and the orientation of the eyes. Nature (London), 1965, 207, 36-37.

Rodivck, R. W. The vertebrate retina. San Francisco: Freeman, 1973.

Shortess, G. K. Some comments on the functional significance of centrifugal fibers to the vertebrate retina. In J. C. Armington, J. Krauskopf, \& B. R. Wooten (Eds.), Visual psychophysics and physiology. New York: Academic Press, 1973.

Sulg, I. A., Hokmanen, E., Sirvola, J., \& Reunanen, M. The electroretinogram, visual evoked cortical potential and retinocortical activation time. In C. Barber (Ed.), Evoked potentials. Baltimore, Md: University Park Press, 1980.

VAN VoORhis, S., \& Hillyard, S. Visual evoked potentials and selective attention to points in space. Perception \& Psychophysics, 1977,22, 54-62.

(Received for publication September 17, 1982; revision accepted December 20,1982.) 